\title{
A115 IMPLICATION OF IDO IN RA PATIENTS TREATED BY BIOLOGICS
}

Guillot X, Rullé S, Mussard J, Falgarone G EA4222, Li2P and Rheumatology Department, Paris 13 University Bobigny, and AP-HP, France

\subsection{6/ard.2010.148981.18}

Indoleamine 2-3 dioxygenase (IDO) is an enzyme involved in immune tolerance, which is thought to be defective in rheumatoid arthritis (RA). It is expressed in mononuclear cells including dendritic cells (DC), and could play a role in DC regulatory $\mathrm{T}$ Cells crosstalk. The authors' aim was to study IDO expression in peripheral blood of RA patients compared to osteoarthritis (OA) and ankylosing spondylitis (AS) controls. The authors also tried to induce IDO expression in vitro, in PBMC from biologic treated patients.

RA patients who fulfilled ACR criteria were included if they were scheduled for biologics. Baseline characteristics of patients were listed and the therapeutic response was evaluated according to EULAR criteria. Human PBMC were purified by density gradient centrifugation, then IDO gene expression was assessed using q-RTPCR; in parallel the kynurenine dosage was performed in plasma to evaluate IDO activity. For some, PBMC were cultured for $24 \mathrm{~h}$ with LPS, IFN- $\gamma$ or LPS + IFN- $\boldsymbol{\gamma}$ before IDO assays.

The authors included 37 RA patients and 20 controls including $9 \mathrm{AS}$ and $11 \mathrm{OA}$. The results showed that IDO was over expressed and more active in RA patients compared to noninflammatory patients. Kynurenine levels were significantly higher in RA patients compared to all control groups In RA patients, before treatment, kynurenine plasmatic level was negatively correlated with DAS28 activity score $(r=-0.552$; $\mathrm{p}<0.016)$, and IDO $\mathrm{mRNA}$ ratio with erythrocyte sedimentation rate $(r=-0.536 ; \mathrm{p}<0.0034)$. After 3 months of biologics (whatever drugs used), kynurenine levels significantly decreased in responders, while they remained unchanged in non-responders. In culture assays, IDO mRNA ratio and kynurenine in supernatants strongly increased after IFN- $\gamma$ and LPS + IFN- $\gamma$ stimulation in RA patients, and to a lesser extent in controls only after LPS ${ }^{+}$IFN- $\gamma$ stimulation. In RA patients treated with anti-tumour necrosis factor $(n=8)$, IDO mRNA ratio significantly decreased after 1 and 3 months. In patients treated with tocilizumab (RIL6-inhibitor, n=14), IDO mRNA expression was downregulated after 1,2 and 3 months of treatment, and kynurenine levels were significantly lower after 3 months of treatment $(n=7)$. Abatacept (CTLA4-Ig; $n=9)$ and rituximab (B cell depletion; $\mathrm{n}=3$ ) did not significantly modified IDO gene expression nor kynurenine levels in RA patients.

In conclusion, IDO is upregulated and more functional in RA patients. It is negatively correlated with systemic inflammation and disease activity, and IDO gene expression is highly inducible. After biologics, IDO function and induction capacities decreased, especially for anti-tumour necrosis factor and anti-RIL6. IDO could contribute to decrease systemic inflammation and disease activity, in a counter-regulation loop that need further experiments. 